\title{
Micro-texturing into DLC/diamond coated molds and dies via high density oxygen plasma etching
}

\author{
Ersyzario Edo Yunata ${ }^{1}$ and Tatsuhiko Aizawa ${ }^{2, *}$ \\ 1 Department of Regional Environment, Shibaura Institute of Technology, 3-9-14 Shibaura, Minato-City, Tokyo 108-8548, Japan \\ 2 Department of Engineering and Design, Shibaura Institute of Technology, 3-9-14 Shibaura, Minato-City, Tokyo 108-8548, Japan
}

Received 23 January 2015 / Accepted 9 June 2015

\begin{abstract}
Diamond-Like Carbon (DLC) and Chemical Vapor Deposition (CVD)-diamond films have been widely utilized not only as a hard protective coating for molds and dies but also as a functional substrate for bio-MEMS/ NEMS. Micro-texturing into these hard coated molds and dies provides a productive tool to duplicate the original mother micro-patterns onto various work materials and to construct any tailored micro-textures for sensors and actuators. In the present paper, the high density oxygen plasma etching method is utilized to make micro-line and microgroove patterns onto the DLC and diamond coatings. Our developing oxygen plasma etching system is introduced together with characterization on the plasma state during etching. In this quantitative plasma diagnosis, both the population of activated species and the electron and ion densities are identified through the emissive light spectroscopy and the Langmuir probe method. In addition, the on-line monitoring of the plasmas helps to describe the etching process. DLC coated WC (Co) specimen is first employed to describe the etching mechanism by the present method. Chemical Vapor Deposition (CVD) diamond coated WC (Co) is also employed to demonstrate the reliable capacity of the present high density oxygen plasma etching. This oxygen plasma etching performance is discussed by comparison of the etching rates.
\end{abstract}

Key words: Micro-texturing, Diamond like carbon, CVD-diamond, Oxygen plasma, Etching, Mold-dies

\section{Introduction}

Diamond-Like Carbon (DLC) and Chemical Vapor Deposition (CVD)-diamond films have been widely utilized as a protective coating of mechanical, functional and fashionoriented parts besides tools and dies [1]. For an example, the boron-doped diamond or nano-crystalline diamond coatings are favored for application to electrically conductive substrates and bio-actuators $[2,3]$. In addition, micro-textures or micropatterns on the metallic or polymer products and tools, work to reduce the friction and wear of their surfaces under the presence of minimum quantity lubrication (MQL) [4, 5]. Hence, the micro-textured DLC- and diamond-coatings are expected not only to further reduce the friction and wear under MQL but also to work as a mold-die to duplicate their micro-textures onto the metallic and polymer sheets even without lubricating oils [6-8].

In order to put the above manufacturing policy into practice, reliable machining methods have to be cultivated as a productive means to make micro-texturing onto the DLCand diamond-coatings. Micro-EDM (electrical discharge

*email: taizawa@sic.shibaura-it.ac.jp machining) is effective to make micro-patterns onto the electrically conductive DLC- and diamond-films [9]. Its geometric accuracy in cutting the films is determined by the diameter of wires in EDM; when the size and dimension of micro-pattern unit becomes less than $100 \mu \mathrm{m}, \mathrm{EDM}$ is difficult to be applied in practice. In addition, large amount of leading time is needed to make machining a thousand or a million of micro-textures onto these films.

Chemical etching by $\mathrm{CF}_{4}+\mathrm{O}_{2}$ mixture gas or lithography is also available to make fine micro-textures with aid of masking [10]. Its slow etching rate by $1.8 \mu \mathrm{m} / \mathrm{h}$ is unsuitable to practical micro-texturing with high aspect ratio. Since $\mathrm{CF}_{4}$ is a typical hazard gas to be post-treated in the closed system to avoid from direct emission in air. The authors have been developing the high density oxygen plasma etching and ashing method for DLC- and diamond-coated tools and dies [11-15]. Since only a pure oxygen gas is used in practice, every etching process can be done in open to air. To be discussed in later, its fast etching rate is also suitable to industrial application. Furthermore, the oxygen plasma state is controllable by using the devices in suitable to each application.

In the present paper, this plasma etching process is applied to make fast-rate micro-texturing of the DLC- and 


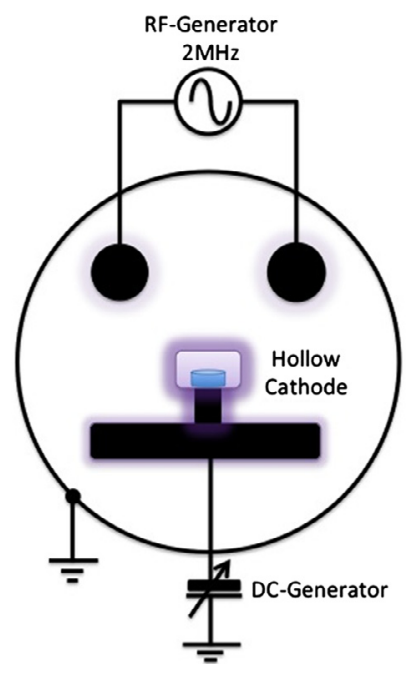

Figure 1. High density oxygen plasma etching system with a hollow cathode.

diamond-coatings. First, a high density oxygen plasma generation is quantitatively discussed by using the plasma diagnosis instrument. Both the emissive-light optical spectroscopy and the Langmuir probe method are utilized for this diagnosis. As a device to control the oxygen ion and electron densities in plasmas, the hollow cathode device is used to investigate the effect of oxygen ion density on the etching behavior.

Two kinds of DLC-coated silicon specimens are first employed to describe the fast-rate and homogeneous etching behavior: e.g. DLC coating with hydrogen content and hydrogen-free DLC. The effect of the hydrogen content in DLC on the etching rate is investigated to describe the etching performance of carbon based coatings. CVD-diamond coated WC (Co) specimens are also used to demonstrate how fine and fast the present oxygen plasma etching makes micro-textures onto the diamond coating.

\section{Experimental procedure}

\subsection{Oxygen plasma etching system}

The high density oxygen plasma etching system consists of the vacuum chamber, the plasma generator, the control unit, the carrier gas supply, and the plasma diagnosis instrument. The chamber is neutral in electricity; RF dipole electrodes and DC-bias work independently to generate RF and DC plasmas, respectively. Hence, the ionized species and activated radicals in the RF plasma are attracted to this DC biased plate with kinetic energy, as illustrated in Figure 1. Either RF-plasma or DC plasma or, both are ignited by switching on either or both on the control panel. In addition, there is no mechanical matching box for RF plasma generation in this system. Both the input and output powers are automatically matched by frequency adjustment around $2 \mathrm{MHz}$. A standard experimental set up is summarized as follows. The base pressure is less than $5 \times 10^{-3} \mathrm{~Pa}$, and pure oxygen gas (purity; 99.99\%) is only used as a carrier gas. RF voltage, DC bias, and pressure are varied in a range from $100 \mathrm{~V}$ to $250 \mathrm{~V}$, from $-400 \mathrm{~V}$ to $-600 \mathrm{~V}$, and from $25 \mathrm{~Pa}$ to $100 \mathrm{~Pa}$, respectively.

A hollow-cathode device is also employed in this system to control the external plasma condition to etching. As shown in Figure 1, the generated RF-plasma is confined in this hollow so that higher ion and electron densities are attained for etching the DLC- and diamond films. In the following plasma diagnosis, both the emissive-light optical spectroscopic (EOS) analyzer and the Langmuir probe are utilized to quantitatively describe the plasma state for etching. Figure 2a depicts the EOS system to detect the population of activated species in the plasma. With comparison to the reference data, each activated species is identified by using the peak position and intensity, respectively. On the other hand, the electron and oxygen ion densities as well as the electron temperature are measured by using the Langmuir probe in Figure 2b. The generated plasma state is characterized by the variation of electron and ion densities with the pressure in plasmas. In particular, the measured densities by the Langmuir probe method are compared between the oxygen plasmas with and without the hollow cathode.

\subsection{Specimens}

Two types of DLC-coated silicon specimens were respectively prepared by RF-sputtering and un-balanced magnetron sputtering to have $5 \mu \mathrm{m}$ in thickness: the hydrogen-terminated DLC film and the hydrogen free one. Both are symbolized by $\mathrm{a}-\mathrm{C}: \mathrm{H}$ and a-C, respectively in the following. The diamondcoated WC (Co) specimens are also prepared by CVD processing to have $20 \mu \mathrm{m}$ thick diamond coating.

In the following micro-texturing onto DLC-coatings, twotypes of resin-type masking patterns were prepared: the micro-lattice pattern with the line-space of $3 \mu \mathrm{m}$ and the line-and space masking pattern, as shown in Figure 3. The former was utilized to describe the difference in micro-texturing process between a-C:H and a-C films with and without use of the hollow cathode. The latter is used to describe the fine micro-texturing into a-C films.

Next, a stainless sheet mask with the thickness of $50 \mu \mathrm{m}$ is first used for masking on the diamond-coated specimens to make micro-line patterning with the width of $100 \mu \mathrm{m}$.

\subsection{Observation and measurement}

Optical microscope and scanning electron microscope (SEM) were used to observe the etched surface. The surface profilometer (Keyence, Co. Ltd.) and the laser-microscope (Nicon, Co. Ltd.) were employed to measure the surface profile of etched micro-texture. Raman spectroscopy (Renishaw, Co. Ltd.) was also utilized to prove the perfect removal of DLC and diamond coatings from mold substrates.

\section{Experimental results}

\subsection{Plasma diagnosis}

Both the emissive light spectroscopy and the Langmuir probe method were utilized to analyze the generated oxygen 
a)

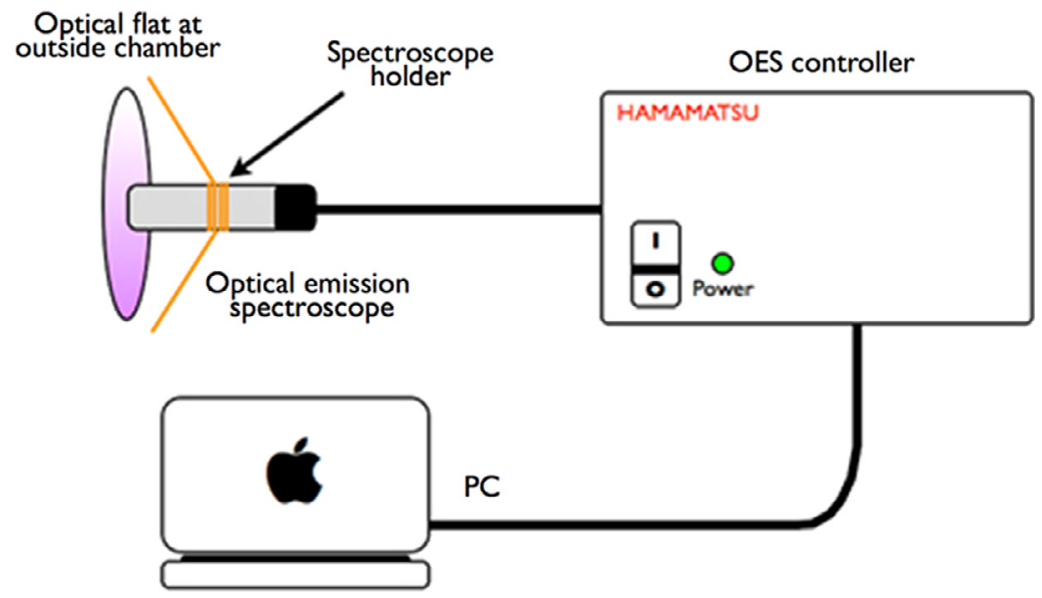

b)

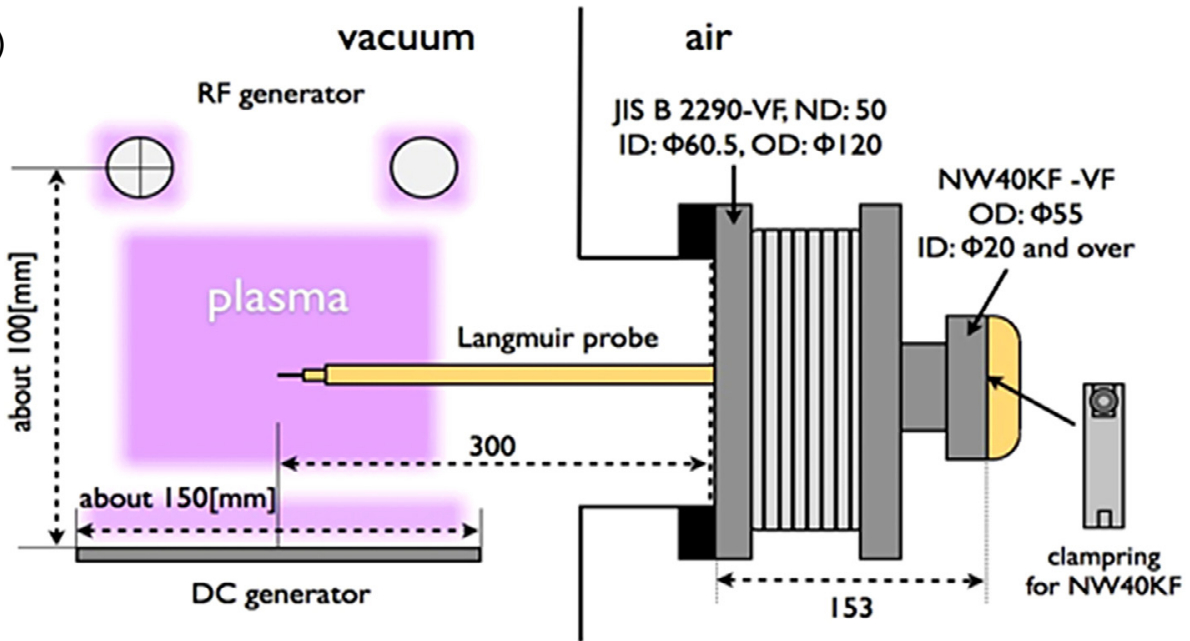

Figure 2. Quantitative plasma diagnosis instrumentation equipped to the high density plasma etching system. (a) Langmuir probe method and (b) emissive-light optical spectroscopy.

plasma state with and without the hollow cathode. Figure 4 compares the emissive light spectra from plasmas with and without the hollow cathode. The oxygen ions $\left\{\mathrm{O}^{+}, \mathrm{O}^{2+}, \mathrm{O}^{3+}\right\}$ or $\{\mathrm{OII}, \mathrm{OIII}, \mathrm{OIV}\}$ and activated oxygen molecules $\mathrm{O}_{2}{ }^{+}$are seen in the spectrum without the hollow cathode, besides the activated oxygen atom $\mathrm{O}^{*}$ or OI. When using the hollow cathode, two strong peaks are seen in the spectrum: OI peaks are mainly observed at $\lambda=776.34 \mathrm{~nm}$ and $843.78 \mathrm{~nm}$, respectively. This reveals that much amount of oxygen atoms are available for etching by using the hollow cathode oxygen plasma.

These two peaks in Figure 4 correspond to the oxygen atom transition $\mathrm{O}\left(3 \mathrm{p}^{5} \mathrm{P} \rightarrow 3 \mathrm{~s}^{5} \mathrm{~S}\right)$ and $\mathrm{O}\left(3 \mathrm{p}^{3} \mathrm{P} \rightarrow 3 \mathrm{~s}^{3} \mathrm{~S}\right)$, respectively. The peak intensity of activated oxygen atoms at $776.34 \mathrm{~nm}$ without the hollow cathode is only $2.5 \times 10^{3}$ counts; while it reaches to around $1.5 \times 10^{4}$ counts with use of the hollow cathode. The high intensity indicates the activated oxygen atom is a main species in the generated oxygen plasma and that its population is enhanced by using the hollow cathode. That is, the oxygen atom flux to the specimen on the cathode becomes lower without the hollow cathode, since the ionization process takes place in all area of the chamber. On the other hand, the ionization advances only in the hollow tube; this confinement of plasmas results in higher oxygen atom flux to the specimen. Next, this difference of plasma state is described by using the Langmuir probe.

Figure 5 compares the variation of electron and ion densities or $\mathrm{Ne}$ and $\mathrm{Ni}$ with the pressure, $p$, in the oxygen plasmas with and without the hollow cathode. In cases without the hollow cathode, $\mathrm{Ne}$ increases and $\mathrm{Ni}$ decreases significantly with increasing the pressure. For an example, $\mathrm{Ne}=1.35 \times$ $10^{15} \mathrm{~m}^{-3}$ and $\mathrm{Ni}=9.60 \times 10^{16} \mathrm{~m}^{-3}$ at $p=65 \mathrm{~Pa}$ while $\mathrm{Ne}=3.30 \times 10^{16} \mathrm{~m}^{-3}$ and $\mathrm{Ni}=3.00 \times 10^{16} \mathrm{~m}^{-3}$ at $p=$ $105 \mathrm{~Pa}$. This pressure dependency of $\mathrm{Ni}$ and $\mathrm{Ne}$ is common to the high density plasmas, irrespective of the cathode configuration. On the other hand, using the hollow cathode, both $\mathrm{Ni}$ and $\mathrm{Ne}$ become higher than those without the hollow cathode. For an example, $\mathrm{Ne}=1.07 \times 10^{16} \mathrm{~m}^{-3}$ and $\mathrm{Ni}=6.99 \times$ $10^{17} \mathrm{~m}^{-3}$ at $p=65 \mathrm{~Pa}$, and, $\mathrm{Ne}=7.51 \times 10^{16} \mathrm{~m}^{-3}$ and $\mathrm{Ni}=1.41 \times 10^{17} \mathrm{~m}^{-3}$ at $p=105 \mathrm{~Pa}$.

The hollow cathode effect on the increase of electron and ion densities is caused by confinement of plasmas in the hollow 

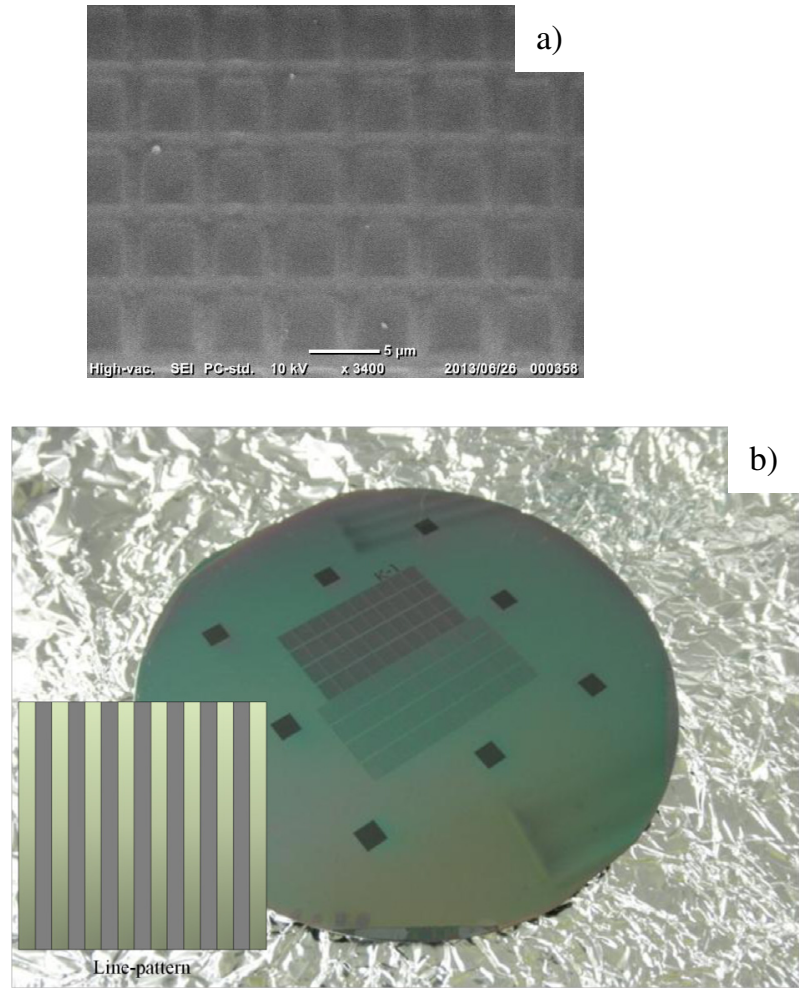

Line-pattern

Figure 3. Two types of resin-type masking patterns for etching the DLC-coated silicon substrates. (a) SEM image of micro lattice patterns with the width of $3 \mu \mathrm{m}$ and (b) micro-line and space patterns for various line widths.

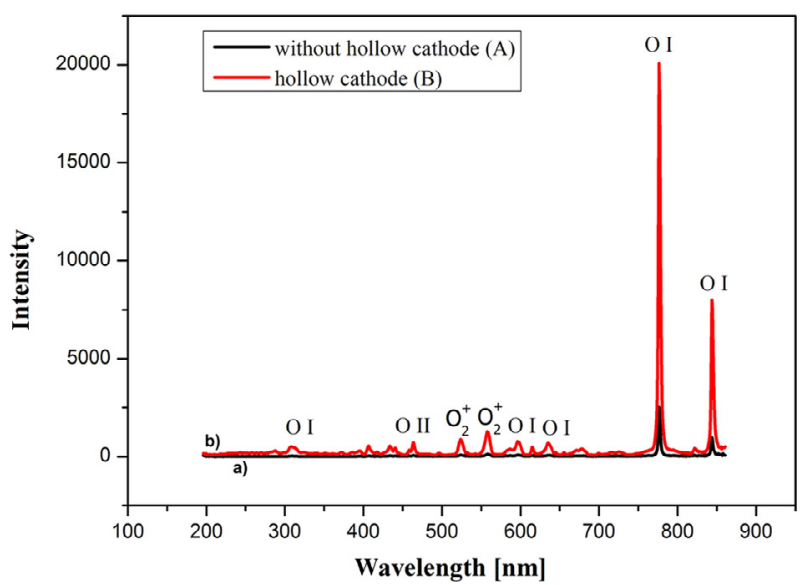

Figure 4. Comparison of the emissive light spectra from plasmas without and with the hollow cathode.

cathode. Electrons in the plasmas have more reaction crosssection with the oxygen molecules to generate more population of oxygen ions. This results in densification both in the electron and oxygen ion state. In the following experiments, this effect of oxygen ion density in plasmas on the etching behavior is experimentally investigated to demonstrate that densification in plasmas is needed for fast-rate etching of DLC- and CVDdiamond coatings.

\subsection{Micro-texturing into DLC-coatings}

Two DLC-coated silicon specimens were employed to describe the difference in etching behavior at the same processing conditions: a-C:H film with the hydrogen content of 10 15 at $\%$, and a-C, hydrogen free DLC-film. The resin-masking was commonly used to make lattice pattern. The film thickness is constant, $5 \mu \mathrm{m}$. Figure 6 compares the etched a-C:H films after etching with and without use of the hollow cathodes after $500 \mathrm{~s}$.

The average etching rate becomes $1.9 \mu \mathrm{m} / \mathrm{h}$ without the hollow cathode and $2.1 \mu \mathrm{m} / \mathrm{h}$ with the hollow cathode, respectively. Since the resin mask has insufficient toughness against the oxygen atom flux, the corners of square patterns are severely over-etched to be round. Next, a-C film is also etched by the hollow cathode method under the same condition as in the above. The average etching rate reaches to $9.2 \mu \mathrm{m} / \mathrm{h}$.

Finer etched surfaces are attained when hollow-cathode etching of a-C films. Then, the micro-line masking pattern with the width of $7 \mu \mathrm{m}$ was used to investigate the etching profile in depth of this a-C films. Figure 7 depicts the SEM image of micro-grooves, etched into the depth of a-C film for $3 \mathrm{ks}$, with the skewed angle of $30^{\circ}$. Since the un-masked a-C film is completely etched away, fine rectangular micro-grooves are formed to have sharp edges with some over-etching. Both the etched bottom and top surfaces are shaped flat with the depth of $4.8 \mu \mathrm{m}$, which corresponds to the a-C film thickness. This proves that the present oxygen plasma etching advances anisotropically into the depth of a-C films.

\subsection{Micro-texturing into CVD-diamond coatings}

CVD-diamond coated WC (Co) specimen was used for oxygen plasma etching with use of the hollow cathode. In this case, the stainless steel sheet with the micro-line patterns was employed as a mask; e.g. the line width was $100 \mu \mathrm{m}$ and the mask width, $20 \mu \mathrm{m}$. The CVD-diamond film is $20 \mu \mathrm{m}$ thick. The experimental conditions are listed in the following: $\mathrm{RF}$ $(250 \mathrm{~V}), \mathrm{DC}(-550 \mathrm{~V})$ and $\mathrm{P}(30 \mathrm{~Pa})$.

The etched diamond-film after $7.2 \mathrm{ks}$ is shown in Figure 8. The unmasked CVD-diamond film is selectively etched away. Different from the etching process of DLC films with use of the resin masking, no over-etching is seen but a sharp edge is formed at the border between the masked and unmasked regions of diamond film. The surface profile in Figure 8b reveals that the gradient of sharp edge by the present etching could be estimated by $\tan (\theta)=20 \mu \mathrm{m} / 4 \mu \mathrm{m} ; \theta=80^{\circ}$. Considering that the metal-mask thickness of $50 \mu \mathrm{m}$ and the clearance between the mask and the diamond film, this edge sharpness is favored for practical micro-patterning into the diamond coatings.

In this rectangular trench with $100 \times 20 \mu \mathrm{m}^{2}$ in Figure 8, its bottom surface turns to be bare WC (Co) substrate surface since almost of all the diamond films are removed away to form this trench or micro-groove. In fact, the measured 
(a)

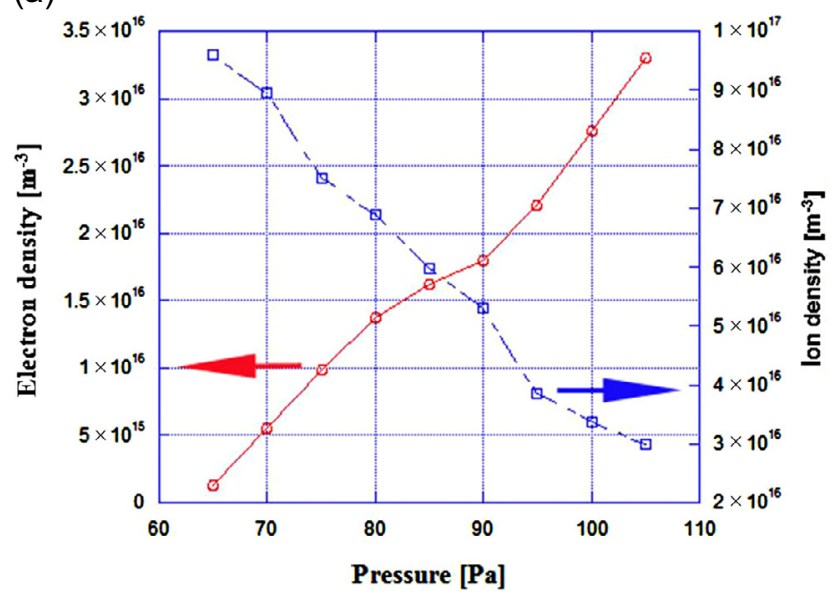

(b)

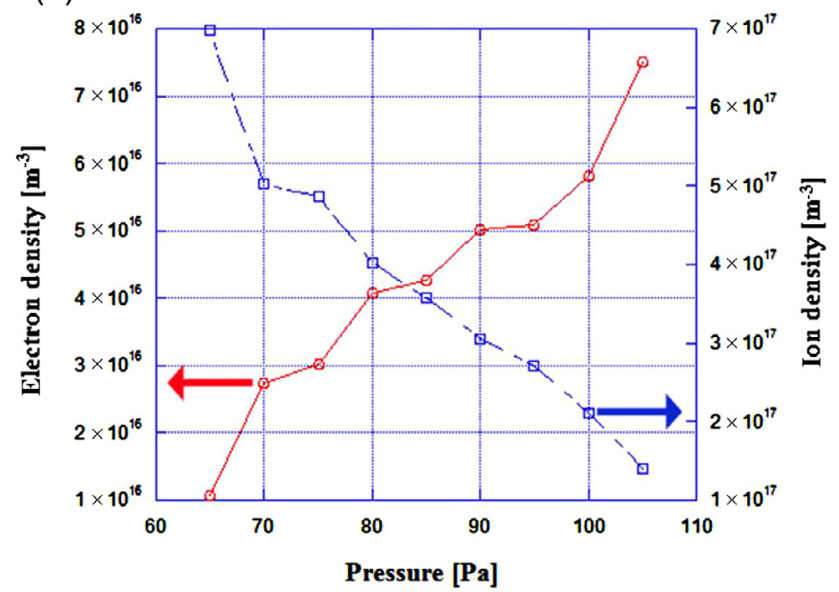

Figure 5. Comparison on the variation of electron and ion densities with the pressure in the plasmas without and with the hollow cathode. (a) without the hollow cathode and (b) with the hollow cathode.
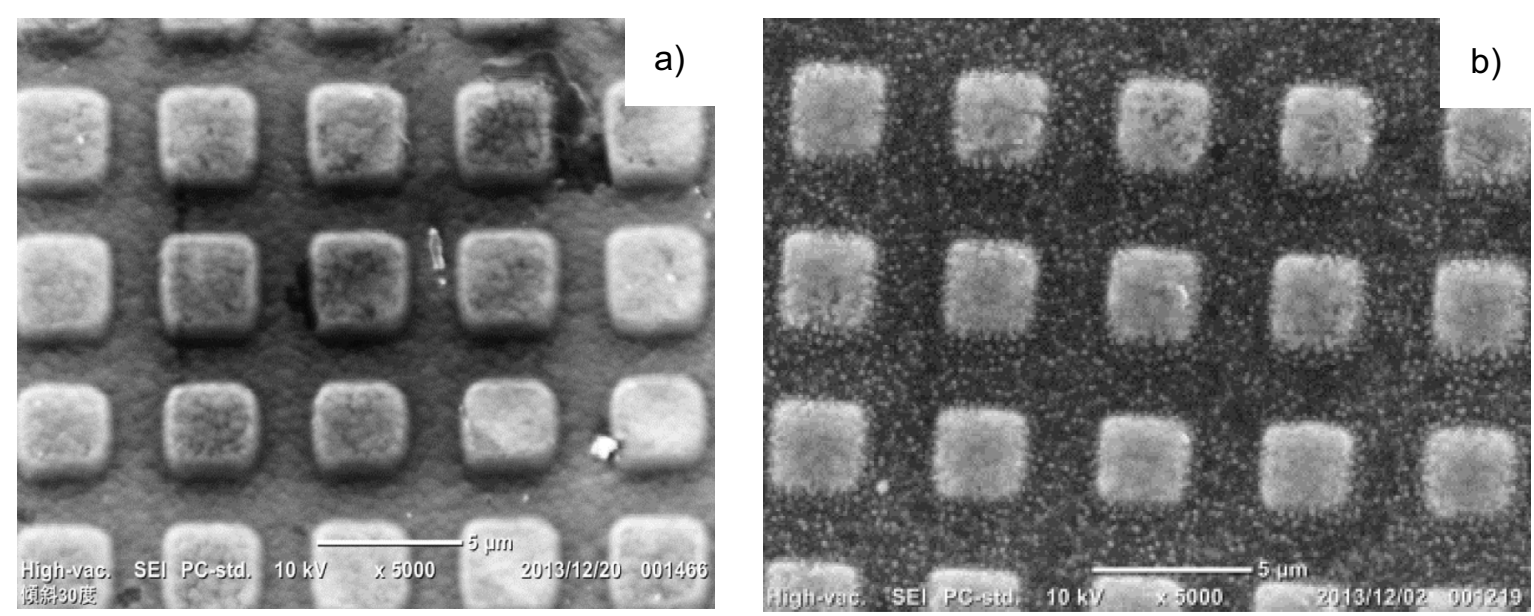

Figure 6. Comparison of SEM images foe etched a-C:H films with and without the hollow cathode. (a) Without the hollow cathode and (b) With the hollow cathode.

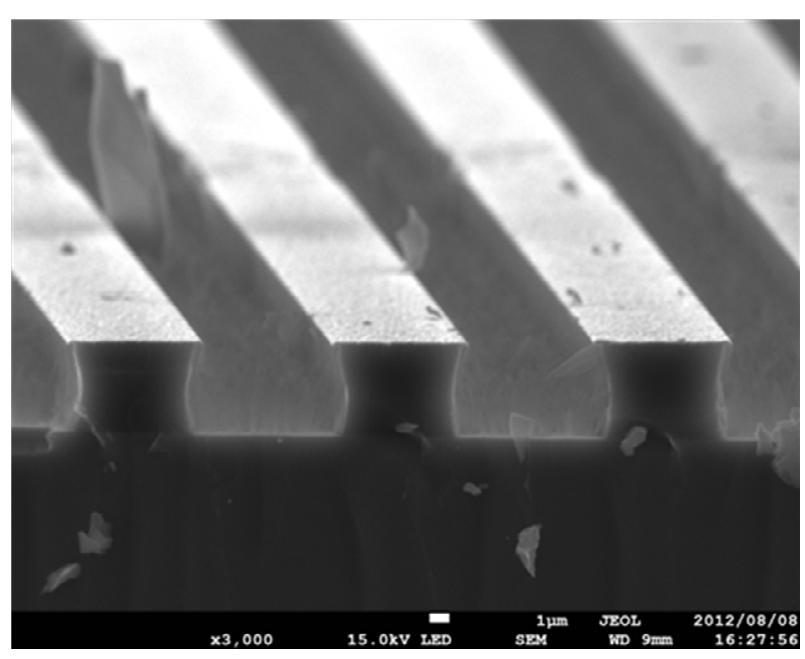

Figure 7. SEM image of etched micro-grooves into the a-C coated silicon substrate with use of micro-line pattern with the line width of $7 \mu \mathrm{m}$. hardness on this bottom surface is nearly constant by $2000 \mathrm{Hv}$, which is equivalent to the original hardness of WC (Co). On the other hand, the masked top surfaces are still diamond coating. This implies that diamond lines are formed by complete ashing of un-masked regions.

\section{Discussion}

Different from the plasma enhanced chemical etching by $\mathrm{CF}_{4}+\mathrm{O}_{2}$, the etching process only by $\mathrm{O}_{2}$ is driven by chemical reaction and physical bombardment. In the former, direct oxidation of carbon based films by C (in DLC and diamond films) $+\mathrm{O}$ (in plasmas) $\rightarrow \mathrm{CO}$ (carbon mono-oxide) is responsible for etching. Hence, if this direct oxidation process were retarded by another chemical reaction, the etching rate could be lowered. In the latter, the physical bombardment effect in etching is enhanced by increasing the DC-bias to accelerate the flux of oxygen ions onto the specimen surface. In particular, the confined plasmas in the hollow-cathode 


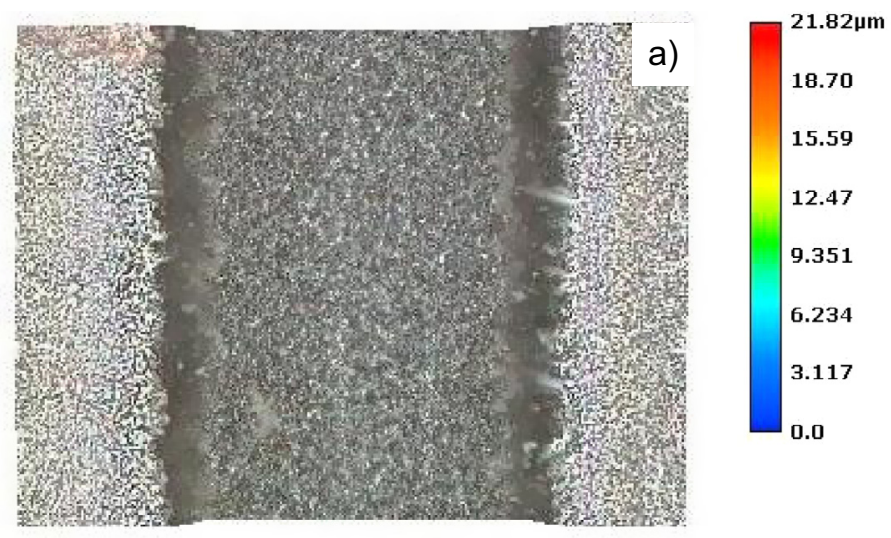

b)

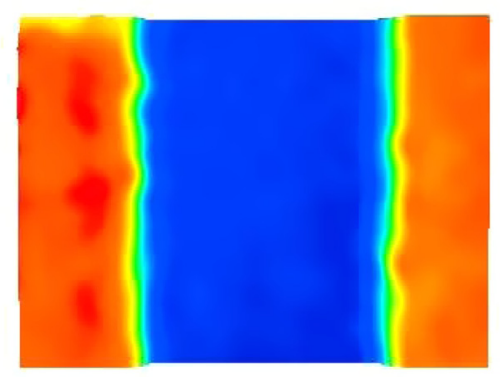

Figure 8. Microgroove formation into the CVD diamond coating by the hollow cathode oxygen plasma etching for 7.2 ks. (a) Optical microscopic image of etched microgroove and (b) surface profile across the microgroove.

device has a capacity to drive the above two etching processes; especially, this device is preferable for anisotropic etching of DLC and diamond coatings. In fact, the etched micro-lattice and micro-groove patterns has the same size and dimension as the original mask patterns. Furthermore, the depth profile of etched micro-patterns is uniform; e.g. the rectangular cross-section of micro-groove is formed by the present etching process.

The hydrogen free DLC with the type of a-C and the CVDdiamond films are etched into micro-textures in fast-rate as predicted by the previous studies in reference [12]. In fact, the etching rates were $9.2 \mu \mathrm{m} / \mathrm{h}$ and $10 \mu \mathrm{m} / \mathrm{h}$ for a-C and diamond coatings, respectively. This high etching performance is driven by the direct oxidation between the sputtered carbon from a-C and diamond and the activated oxygen flux. In application of high DC-bias, the sputtered carbon by the oxygen ions reacts with oxygen atoms at the etching front end; the resultant carbon monoxide is ejected from the formed microtextures. This efficient route of reactions results in higher etching rate for a-C and diamond coatings.

However, in etching the hydrogenated amorphous carbon films, the hollow cathode discharging approach has little influence on the acceleration of etching rate. Using the same resin masks in etching of a-C and a-C:H films with the same thickness, the etching rates were $2.1 \mu \mathrm{m} / \mathrm{h}$ for a-C:H and $9.2 \mu \mathrm{m} / \mathrm{h}$ for a-C. Although the etching rate against a-C:H films is retarded by the resin masking procedure, a relatively high content of hydrogen in a-C:H has possibility to prevent the oxygen atom flux from direct reaction with carbon in a-C:H just like the metal dopes $(\mathrm{M})$ in a-C:H:M.

The hydrogen free a-C type DLC's and CVD-diamond films can be etched in fast-rate as predicted by the previous studies [12]. In particular, higher etching rate of diamond coatings than the conventional etching methods with use of $\mathrm{CF}_{4}$ is preferable for practical applications. As had reported in reference [15], no residuals of diamond films are analyzed to be present by the Raman spectroscopy. Sharp edge formation as well as no residual formation of diamonds by the present etching must be key-technological items in fabrication of diamond bio-MEMS and NEMS.

\section{Conclusion}

The hollow cathode oxygen plasma etching method is proposed as an effective means for etching of DLC- and CVD-diamond coatings. Although the etching process might be retarded by the presence of high content hydrogen in DLC films, fastrate etching is attained by this method both in the hydrogenfree DLC's and CVD-diamond coating. In particular, microtextures are machined into the CVD-diamond coatings with sufficiently sharp edges; i.e. fine rectangular micro-groove is cut into the diamond films with the depth of film thickness. In this approach, the resolution of micro-textures is mainly determined by the geometric accuracy in masking. Various kinds of fine masking are in trial to aim at higher and more precise resolution in this micro-texturing.

\section{Implications and influences}

The present paper has contribution to provide a means for micro-texturing into the CVD diamond coatings via the high density oxygen plasma etching. This method is useful not only for fabrication of dies and molds to duplicate the original micro-textures onto the metallic and polymer sheets but also for construction of sensors and actuators in bio-MEMS and bio-NEMS.

Acknowledgements. The authors would like to express their gratitude to Ms. R. Tsuda (SIT), Mrs. S. Ueda (Fujikoshi, Co. Ltd.), and, Mrs. M. Misawa and Ms. K. Ando (Renishow, Co. Ltd.) for their help in the etching experiments, in CVD-coating and in Raman spectroscopy, respectively. This study is financially supported in part by the MEXT-project with the contract of \# 411419 .

\section{References}

1. A. Erdemir, C. Donnet, Journal of Physics D Applied Physics 39 (2006) 311-327.

2. E. Kohn, P. Gluche, M. Adamschik, Diamond and Related Materials 8 (1999) 934-940. 
3. M.W. Varney, D.M. Aslam, A. Janoudi, H.-Y. Chan, D.H. Wang, Biosensors 1 (2011) 118-133.

4. N. Kawasegi, H. Sugimori, H. Morimoto, N. Morita, I. Hori, Precise Engineering 33 (2009) 248-254.

5. T. Enomoto, T. Sugihara, Manufactruring Technology 59 (2010) 597-600.

6. T. Aizawa, Applied Mechanics and Materials 289 (2013) 23-37.

7. T. Aizawa, T. Fukuda, Research Report of Shibaura Institute of Technology 57 (2013) 1-10.

8. T. Aizawa, T. Fukuda, Surface and Coating Technology 215 (2013) 364-368.

9. P. Agrawal, A. Manoria, R. Thakur, H. Jain, International Journal of Mechanical Engineering and Robotics Research 1 (2012) 121-128.
10. Z. Cao, D.M. Aslam, Proceedings of IEEE on nano-electro mechanical system, Xiamen, China, 2010.

11. T. Aizawa, Proceedings of the 5th Southern East Asia Technology University Consortium Conference, Hanoi, Vietnam, 2011, CD-ROM.

12. T. Aizawa, K. Mizushima, T.N. Redationo, M. Yang, Proceedings of the 8th International Conference on MciroManufacturing 8 (2013) 459-466.

13. T. Aizawa, Y. Sugita, Research Report of Shibaura Institute of Technology 55 (2011) 13-22.

14. T. Aizawa, T. Fukuda, Research Report of Shibaura Institute of Technology 56 (2012) 47-56.

15. E.E. Yunata, T. Aizawa, Manufacturing Letters (2015) (to be published).

Cite this article as: Yunata E.E \& Aizawa T: Micro-texturing into DLC/diamond coated molds and dies via high density oxygen plasma etching. Manufacturing Rev. 2015, 2, 13. 